\title{
Inteligência Emocional: Teoria, Pesquisa, Medida, Aplicações e Controvérsias
}

\author{
Emotional Intelligence: Theory, Research, Measurement, \\ Applications, and Controversies
}

\author{
Carla Woyciekoski \& Claudio Simon Hutz* \\ Universidade Federal do Rio Grande do Sul
}

\begin{abstract}
Resumo
A Inteligência Emocional (IE) constitui um construto psicológico recente, e um dos aspectos da inteligência mais discutidos na atualidade. Ela reflete, sobretudo, o estudo das interações entre emoção e inteligência, o que nos permite inferir a complexidade do campo conceitual, bem como as dificuldades metodológicas daí decorrentes. Este artigo visa informar ao leitor o corrente status da IE do ponto de vista científico, apresentando-se os modelos teóricos correntes desta forma de inteligência, bem como suas principais características, qualidades, falhas, críticas à teoria, aplicações e correlações com outros construtos psicológicos. Além disso, apresenta-se uma discussão acerca dos tipos de instrumentos de medida da IE que têm sido utilizados, incluindo aspectos e pesquisas relacionadas à capacidade destas escalas de predizer comportamentos, de forma independente das medidas psicométricas existentes. Embora sejam notáveis a ampliação do campo e os progressos no desenvolvimento da teoria da IE, bem como dos testes que se propõem medi-la, ainda há problemas a serem sanados e melhor investigados (como os referentes à validade de construto e aos tipos de escalas de IE existentes). Estes e outros aspectos serão relatados e problematizados ao longo do artigo.

Palavras-chave: Inteligência Emocional; inteligência; emoção; personalidade; testes de Inteligência Emocional.

Abstract

Emotional Intelligence (EI) is a recent psychological construct, and also one of the most discussed aspects regarding intelligence. Moreover, it reflects the study of the interrelations between emotion and intelligence, which allows us to infer the complexity of its conceptual field, as well as its methodological difficulties. This article aims to inform the reader of the current status of EI from a scientific point of view. Therefore, it comprises the current EI theoretical models, as well as its main characteristics, qualities, pitfalls, critics, applications and correlations with other psychological constructs. Additionally, it will be discussed the types of instruments that have been used to assess EI, including aspects and researches related to the capacity of these scales to predict behavior, independently of the existing psychometric measures. Although the progress in the development of the EI theory is noticeable, as well as the tests that are devised to measure it, there are still problems remaining unsolved which ought to be better investigated like those related to construct validity and the types of existent scales. These and other aspects will be reported and discussed throughout the article.

Keywords: Emotional Intelligence; intelligence; emotion; personality; Emotional Intelligence tests.
\end{abstract}

Inteligência e emoção são temas que têm instigado pesquisadores e gerado polêmica por mais de um século de estudos e pesquisas (Matthews, Zeidner, \& Roberts, 2002; Siqueira, Barbosa, \& Alves, 1999). A Inteligência Emocional (IE) constitui um campo de investigação relativamente novo, que traz consigo a proposta de ampliar o conceito do que é aceito como tradicionalmente inteligente, incluindo nos domínios da inteligência aspectos relacionados ao mundo das emoções e sentimentos. As concepções atuais sobre inteligência constituem o produto do pensamento, trabalho e investigações de centenas de pesquisadores, que

*Endereço para correspondência: Universidade Federal do Rio Grande do Sul, Instituto de Psicologia, Rua Ramiro Barcelos, 2600, Porto Alegre, RS, Brasil, CEP 90035-003. E-mail: hutzc@terra.com.br ao longo da história, definiram o que é ser inteligente. Assim como atualmente é possível perceber evoluções no pensamento em muitas áreas, também tem sido levantada a necessidade de se repensar o que se entende por inteligência e por comportamento inteligente. A verificação das relações entre cognição e emoção poderia resultar no reconhecimento da capacidade do homem lidar com seu mundo emocional de forma inteligente, compatível com seus objetivos mais amplos de vida.

Na parte inicial deste artigo serão apresentados o histórico e as características gerais da inteligência e da emoção, bem como o contexto histórico do surgimento da IE. Em seguida, serão relatadas as suas principais características, bem como os aspectos relacionados aos diferentes modelos existentes de definição e medição da IE. Além disso, serão abordadas as inter-relações entre a IE e construtos já esta- 
belecidos como personalidade e inteligência, tendo como base as discussões teóricas e pesquisas evidenciadas na literatura. A segunda parte do artigo reservará um espaço para a discussão do status atual da pesquisa em IE, bem como as principais áreas de pesquisa e aplicações práticas, destacando-se os presentes achados relativos à capacidade preditiva da IE. Salienta-se que este é um aspecto fundamental no processo de constituição de um novo construto, especialmente no que se refere à construção da proposta de uma nova forma de inteligência. Por último, serão apresentadas as considerações finais, bem como serão apontados caminhos para pesquisas futuras.

\section{Inteligência: Histórico e Definição}

A partir do século XIX, observou-se um crescente interesse pela inteligência humana, especialmente quando Herbert Spencer e Francis Galton sugeriram uma capacidade humana geral e superior. Galton entendia a inteligência como o reflexo de habilidades sensoriais e perceptivas transmitidas geneticamente. Assim como este, Raymond Cattell também acreditava que testes baseados em habilidades mentais simples (como tempos de reação, discriminação sensorial e associação de palavras) poderiam constituir importantes preditores do desempenho acadêmico. Contudo, estudos posteriores demonstraram que escalas baseadas em habilidades simples não constituíam preditoras de sucesso acadêmico, além de não serem adequadas para medir a inteligência (Carroll, 1982).

Após ter investigado os testes mentais elaborados por estes e outros pesquisadores, Alfred Binet concluiu que escalas que incluíssem capacidades mais complexas e atividades do dia-a-dia seriam mais adequadas para medir a inteligência. Em 1905, ele e Théophile Simon criaram o primeiro teste satisfatório de inteligência, por meio de uma solicitação do Ministério de Educação Francês que objetivava diagnosticar crianças necessitadas de educação especializada (Matthews et al., 2002). A escala Binet-Simon incluía itens que abrangiam a compreensão da linguagem e a habilidade de raciocinar a nível verbal e não verbal. Este teste constituiu a base de pesquisas futuras e foi utilizado em vários países e línguas. Após alguns anos, iniciaram-se as pesquisas em avaliação mental de adultos, especialmente quando em 1939, David Wechsler criou a Escala Wechsler de Inteligência para Adultos (WAIS), também revisada posteriormente.

Com relação a sua definição, é possível perceber duas correntes teóricas. Há autores que a definiram como uma capacidade geral de compreensão e raciocínio, enquanto outros a descreveram como envolvendo diversas capacidades mentais relativamente independentes umas das outras. Binet e Wechsler foram adeptos do primeiro pressuposto. Do mesmo modo, em 1904, Charles Spearman sugeriu a existência de um fator geral de inteligência $(g)$, o qual permearia o desempenho em todas as tarefas intelectuais. Segundo ele, as pessoas seriam mais ou menos inteligentes, dependendo da quantidade de $g$ que possuíam. Spearman estava especialmente interessado na natureza psicológica e na interpretação do componente mental que tende a produzir correlações positivas entre os vários testes. Por meio de vários estudos, ele sugeriu que o $g$ era um fator central e supremo em todas as medidas de inteligência, o qual representava a capacidade de raciocínio ou a gênese do pensamento abstrato (Carroll, 1982; Sternberg, 1992).

Todavia, em 1938, Thurstone criticou a inteligência geral de Spearman, e postulou que a inteligência poderia ser decomposta em várias capacidades básicas através da análise fatorial. Thurstone identificou sete fatores (compreensão verbal, fluência verbal, aptidão numérica, visualização espacial, memória, raciocínio e velocidade perceptiva) e criou o Teste de Capacidades Mentais Básicas (Butcher, 1968/1974). Similarmente, Guilford (1967) propôs que a inteligência compreenderia 150 fatores. Gardner (1995) criou a teoria das Inteligências Múltiplas, independentes entre si, as quais operariam em blocos separados no cérebro, obedecendo a regras próprias: inteligência lógico-matemática, lingüística, musical, espacial, corporal-cinestésica, intrapessoal e interpessoal.

Neisser et al. (1996) propuseram que as pessoas se diferenciam nas habilidades de compreender idéias complexas, de se adaptarem ao ambiente, de aprender com a experiência, na maneira como conduzem seu raciocínio e resolvem problemas através do pensamento. No entanto, mesmo que estas diferenças individuais sejam substanciais, elas raramente são consistentes, uma vez que a performance intelectual de determinada pessoa varia em ocasiões e domínios diferentes. Sendo assim, muitos teóricos da atua-lidade (Campione, Brown, \& Ferrara, 1982; Gardner, 1995; Mayer \& Salovey, 1997) sugeriram a existência de muitas inteligências, as quais constituiriam sistemas de habilidades. Num documento emitido pela American Psychological Association (APA), enfatizou-se que pouco se sabe sobre as possíveis formas de inteligência, e que os testes atuais seriam capazes de captar apenas algumas destas inteligências, sugerindo a existência de outras, as quais teriam sido bem menos estudadas e compreendidas (APA, 1997). Conforme Campione et al. (1982), a inteligência acadêmica constituiria uma das formas de inteligência possíveis (não a única).

Em 1997, Sternberg salientou que uma das características mais importantes da inteligência seria a capacidade de pensar de forma abstrata. Seguindo esta premissa, Mayer, Salovey, Caruso e Sitarenios (2001) ressaltaram que o raciocínio abstrato somente seria possível através de um input ou entrada de um estímulo (informação) no sistema, de modo que diferentes inteligências seriam definidas de acordo com o que entra e é processado no sistema. Os autores argumentaram que a informação ingressante poderia ser de ordem verbal, espacial, social e emocional, entre outras.

Uma das primeiras tentativas de ampliar o conceito de inteligência para além de capacidades intelectuais gerais (usualmente relacionadas a competências acadêmicas) foi conduzida por iniciativa de Thorndike (1936). Ele propôs a Inteligência Social (IS), como a capacidade de perceber os estados emocionais próprios e alheios, motivos e com- 
Woyciekoski, C. \& Hutz, C. S. (2009). Inteligência Emocional: Teoria, Pesquisa, Medida, Aplicações e Controvérsias.

portamentos, além da capacidade de agir com base nestas informações de forma ótima. Sobretudo, a IS refletiria a habilidade de decodificar informações oriundas do contexto social e de desenvolver estratégias comportamentais eficazes com vistas a objetivos sociais (Siqueira et al., 1999). Sternberg e Salter (1982) já haviam referido que grande parte da inteligência consiste em resolver uma variedade de problemas apresentados nos diferentes contextos sociais. Sternberg (1997) argumentou que os seres humanos são essencialmente sociais; e a ausência de habilidades sociais poderia significar uma limitação importante na capacidade de adaptação social bem sucedida. Assim como reconhece-se a importância destas habilidades, cabe destacar o papel das emoções na adaptação social e no comportamento inteligente.

\section{Emoção}

Conforme salientaram Matthews et al. (2002), para que possamos compreender a IE é necessário que se tenha claro a concepção de emoção. Além disso, o próprio aspecto multidimensional das emoções ocasionaria uma concepção de IE complexa. Segundo Fortes D’Andrea (1996), poucos fatos psicológicos se comparam com as emoções, pois elas demarcam fatos importantes em nossa vida, mas mais do que isso, elas influenciam a forma como reagimos a estas experiências. Smith e Lazarus (1990) argumentaram que elas podem causar importantes impactos no bemestar subjetivo das pessoas, na saúde física e mental, nas interações sociais, além de influenciar a capacidade de resolução de problemas. Campos, Campos e Barret (1989, citados em Garber \& Dodge, 1991) sugeriram que as emoções seriam responsáveis pelas relações da pessoa com o ambiente externo, bem como pela sua manutenção ou interrupção. Para estes autores a coordenação de múltiplos processos é uma característica principal da emoção.

Sendo assim, a emoção corresponderia a uma reação psicobiológica complexa, que envolveria inteligência e motivação, impulso para ação, além de aspectos sociais e da personalidade, que acompanhados de mudanças fisiológicas, expressariam um acontecimento significante para o bemestar subjetivo do sujeito no seu encontro com o ambiente. Sob este prisma, a emoção seria parcialmente biologicamente determinada, e parcialmente o produto da experiência e do desenvolvimento humano no contexto sociocultural (Smith \& Lazarus, 1990). Lopes, Brackett, Nezlek, Schütz e Salovey (2004) salientaram que competências emocionais são essenciais nas interações sociais porque emoções alimentam funções comunicativas e sociais, além de conterem informações sobre os pensamentos e intenções das pessoas. Segundo os autores, a ocorrência de uma interação social positiva e satisfatória demandaria que os indivíduos percebessem, processassem e manejassem a informação emocional de forma inteligente. A visão de que as competências emocionais são cruciais para adaptação tem suscitado o interesse pelo tema da inteligência emocional e inspirado muitos programas de aprendizagem social e emocional em escolas e em ambientes de trabalho.

\section{Inteligência Emocional}

A Inteligência Emocional (IE) constitui um campo em expansão que engloba várias áreas de pesquisa. A concepção da IE como uma habilidade foi desenvolvida numa série de artigos na década de 1990 (Mayer, DiPaolo, \& Salovey, 1990; Salovey \& Mayer, 1990), sendo que a pesquisa inicial visou a aspectos teóricos de delimitação de construto, medição e comprovação empírica, baseados no modelo psicométrico de inteligência (Mayer, Salovey, \& Caruso, 2002). Ela foi definida academicamente pela primeira vez por Salovey e Mayer (1990), como uma subforma de IS que abrangeria a habilidade de monitorar as emoções e sentimentos próprios e dos outros, discriminá-los e utilizar essas informações para orientar pensamentos e ações. Os primeiros estudos empíricos demonstraram a habilidade das pessoas em identificar emoções em cores, rostos e formas (Mayer et al., 1990), além de ter sido investigado a compreensão de emoções de personagens em histórias (Mayer \& Geher, 1996).

Entre 1994 e 1997 procedeu-se o fenômeno da popularização da IE, especialmente quando Daniel Goleman (1996), lançou o livro intitulado "Emotional intelligence", ocasionando a ampliação e a "mudança" da definição da IE (em especial na mídia e literatura popular), que a partir de então passou a incluir aspectos da personalidade. A reação à popularização, por parte dos proponentes da IE (Mayer \& Salovey, 1997) foi de questionar expectativas infundadas, além de terem redefinido a IE:

A inteligência emocional envolve a capacidade de perceber acuradamente, de avaliar e de expressar emoções; a capacidade de perceber e/ou gerar sentimentos quando eles facilitam o pensamento; a capacidade de compreender a emoção e o conhecimento emocional; e a capacidade de controlar emoções para promover o crescimento emocional e intelectual. (Mayer \& Salovey, 1997, p. 15).

A partir desta revisão, o processamento de informações emocionais foi explicado através de um modelo de quatro níveis: (a) percepção acurada das emoções; (b) uso da emoção para facilitar pensamento, resolução de problemas e criatividade; (c) compreensão de emoções; e (d) controle de emoções para crescimento pessoal (Mayer et al., 2002). Segundo os autores, a Percepção Emocional (PE) constituiria a mais básica das habilidades da IE, a qual refletiria a aptidão para reconhecer distintas emoções em si e nos outros de maneira acurada, além da capacidade de expressálas nas situações sociais. A PE poderia estar associada a um sentimento de competência para lidar com diferentes situações e pessoas, na medida em que o componente emocional poderia agir como um importante recurso de informação. A Emoção como Facilitadora do Pensamento refere-se à capacidade de o pensamento gerar emoções, bem como a possibilidade das mesmas influenciarem o processo cognitivo. Conforme Forgas (1995) e Schwarz (1990), emoções poderiam influenciar processos de pensamento por meio da promoção de distintas estratégias de processamento da informação. Pessoas hábeis em integrar suas emoções 
com a cognição, tenderiam a utilizar emoções positivas para desenvolver criatividade e processar a informação de forma integrada. Além disso, estas pessoas necessitariam de menor esforço cognitivo no processamento de informação e na resolução de problemas de ordem emocional (Schwarz, 1990).

A capacidade de Compreensão Emocional (CE) estaria relacionada a três habilidades: (a) capacidade de identificar emoções e codificá-las; (b) entender os seus significados, curso e a maneira como se constituem e se correlacionam; e (c) conhecer suas causas e conseqüências. Adicionalmente, a CE indicaria o quão bem uma pessoa seria capaz de entender significados e situações emocionais, através da utilização de processos de memória e codificação emocional (Mayer, Salovey, \& Caruso, 2002, 2004a). Gohm, Corser e Dalsky (2005) e Lyons e Schneider (2005) referiram que a capacidade de entender e prever novas emoções poderia estar associada a sentimentos de previsão e controle. O Gerenciamento Emocional $(G E)$ refletiria à capacidade de regular emoções em si e nos outros, isto é, de gerar emoções positivas e reduzir as negativas, conforme o caso (Mayer \& Salovey, 1997). Pessoas hábeis em modificar as emoções de forma a modelar respostas afetivas de acordo com seus objetivos e com o meio, poderiam obter benefícios em variadas situações, como de estresse, por exemplo (Lyons \& Schneider, 2005). Igualmente, a possibilidade de reduzir emoções intensas e de gerar experiências emocionais poderia ocasionar sentimentos de auto-controle. A habilidade de regular as emoções nos outros poderia ocasionar sentimentos de controle situacional. Além disso, GE foi relacionado à satisfação com a qualidade das interações sociais, bem como com uma tendência a obter suporte dessas (Lopes, Salovey, \& Straus, 2003). Sobretudo, o controle emocional traduziria a habilidade de regular emoções com o objetivo de promover bem-estar e crescimento emocional e intelectual.

\section{Critérios para Padronização da IE: Dois Modelos Teóricos}

Mayer, Caruso e Salovey (2000) referiram que a IE requer o cumprimento de três critérios rigorosos para atingir o status de uma inteligência como as já estabelecidas: conceitual, correlacional e desenvolvimental. O primeiro associa-se à necessidade de a IE refletir uma performance mental ao invés de formas de comportamento, auto-estima, ou características não intelectivas, sendo que as habilidades relacionadas às emoções devem ser medidas através de testes que requeiram desempenho mental. O segundo critério, que descreve padrões empíricos, traduz-se pela necessidade da IE abranger um conjunto de habilidades relacionadas que seriam similares, mas distintas das habilidades mentais descritas por inteligências previamente existentes (Neisser et al., 1996). O terceiro critério postula que a inteligência deve ser passível de aprimoramento ao longo da vida (com a idade e experiência).

Conforme mencionado anteriormente, embora a IE tenha sido concebida como uma habilidade, que abrange o processamento cognitivo da informação afetiva e emocional, ela também foi definida como envolvendo muito mais que habilidades como perceber, assimilar, entender e gerenciar emoções. Foi assim que surgiram as concepções alternativas da IE, como os modelos propostos por Bar-On (1997) e Goleman (1996), os quais possuem campos de definição vastos, como motivação e dimensões de personalidade, tais como, persistência, zelo e otimismo (Goleman, 1996; Schutte et al., 1998). Mayer, Caruso, et al. (2000) denominaram estas concepções de mistas, por incluírem nas suas definições conceitos não intelectivos. Goleman (1996), por exemplo, afirmou que a IE envolveria autoconsciência, empatia, autocontrole, sociabilidade, zelo, persistência e auto-motivação. Referiu-se à IE como caráter, e sugeriu que ela determinaria em grande parte o sucesso ou o fracasso das relações e experiências cotidianas. Em outro livro, afirmou que a IE é responsável por cerca de $85 \%$ do desempenho de líderes bem sucedidos, ou que comparada com o QI, a IE é duas vezes mais importante (Goleman, 1998). Mais tarde, entretanto, o próprio Goleman (2000, p. 22, citado por Mayer et al., 2004a) teria reconhecido a necessidade de mais pesquisa acerca de tais afirmações.

Uma das maiores contribuições de Mayer, Salovey e Caruso (2000) para o campo de investigação da IE, referese ao fato de que sua teoria não se baseia em promessas incomuns relacionadas ao potencial da IE. Estes pesquisadores têm procurado expor as promessas e ditos populares referentes à IE como infundados, visto a falta de evidências capazes de sustentar tais afirmações. Eles enfatizaram que cientistas acadêmicos deveriam discernir entre senso comum e pesquisa científica. Contrariamente a sobreposição de conceitos corrente nas concepções mistas da IE, pesquisas têm apoiado a existência da IE como uma habilidade mental (assim como a definem Mayer, Caruso, et al., 2000). A teoria prediz que a IE é uma inteligência como outras existentes, pois ela comporta três critérios empíricos: (a) problemas emocionais têm respostas certas ou erradas avaliadas por métodos de escores alternativos (por consenso do grupo, por especialistas ou por consulta ao alvo, isto é à pessoa que expressou emoções a serem avaliadas em determinado teste); (b) as habilidades medidas se correlacionam com outros testes de habilidades mentais, e (c) o nível da habilidade aumenta com a idade (Mayer, Salovey, et al., 2000).

\section{Instrumentos de Medida da IE}

Conforme apontaram Brackett e Mayer (2003), a pesquisa em IE expandiu-se na última década e atualmente conta com inúmeros instrumentos de avaliação. Contudo, o campo da IE tem-se caracterizado por dificuldades de mensuração, devido aos problemas teóricos de delimitação de construto e devido aos tipos de instrumentos utilizados para medir essa aptidão. Comumente, a IE é medida através de instrumentos de avaliação de dois tipos: (a) os de desempenho, que medem a performance de determinado sujeito em tarefas específicas e (b) os de auto-relato, que 
Woyciekoski, C. \& Hutz, C. S. (2009). Inteligência Emocional: Teoria, Pesquisa, Medida, Aplicações e Controvérsias.

constituem questionários onde o sujeito reporta as habilidades que acredita possuir. Atualmente, os mais renomados testes disponíveis internacionalmente são o Mayer-SaloveyCaruso Emotinal Intelligence Test (MSCEIT) (Mayer et al., 2002), o Emotional Quotient Inventory (EQ-i) (Bar-On, 1997), e o Schutte Self-Report Inventory (SSRI) de Schutte et al. (1998), sendo as duas últimas escalas de auto-relato. No Brasil, dispomos de um instrumento baseado em autorelato, a Medida de Inteligência Emocional (MIE) (Siqueira et al., 1999) e a escala de Percepção de Emoções do MEIS (Mayer Emotional Intelligence Scale), uma versão anterior ao MSCEIT, validada para o Brasil por Bueno e Primi (2003). Além disso, pesquisas com o MSCEIT traduzido estão sendo desenvolvidas no Brasil por estes últimos autores e outros.

Embora tenham sido propostas diferentes teorias da IE, há controvérsia na comunidade acadêmica sobre como a IE deve ser definida e medida. Mayer et al. (2002) propuseram que pesquisadores deveriam definir a IE por meio de habilidades ao invés de dimensões da personalidade, e argumentaram que isso seria necessário para que se pudesse assegurar a validade discriminante em relação a construtos de personalidade, por exemplo. Os autores sugeriram o uso de testes de desempenho ao invés de testes de auto-relato, visto que escalas de performance poderiam medir algo mais diretamente relacionado à capacidade de pensamento abstrato e de gerar pensamentos sobre emoções. Além disso, defenderam que testes de desempenho dificultariam a simulação de desempenho, além de evitar avaliações subjetivas acerca das habilidades emocionais.

Em contrapartida, outros autores definiram o construto como uma disposição (assim como as dimensões de personalidade), a qual poderia ser medida através de instrumentos de auto-relato (Bar-On, 1997; Goleman, 1996; Schutte et al., 1998). Entretanto, muitos autores afirmaram que auto-relatos não medem habilidades emocionais. Brackett e Mayer (2003) argumentaram que estas medidas da IE não são confiáveis, uma vez que estaria em jogo a capacidade acurada do indivíduo de reportar suas próprias habilidades. Eles destacaram que, em geral, as pessoas seriam informantes inacurados de suas próprias habilidades. Em concordância, Bueno e Primi (2003) consideraram inadequado medir qualquer tipo de inteligência questionando um indivíduo sobre o quanto ele se considera inteligente. Estes autores também referiram um aspecto muito importante, que aponta para o fato de que a auto-percepção da capacidade de solucionar problemas não se relaciona diretamente a real capacidade de desempenho de um indivíduo. Assim como destacaram Mayer et al. (2004a): "a inteligência auto-referida de uma pessoa é consideravelmente diferente da sua inteligência real” (p. 203). Em uma pesquisa, Brackett e Mayer (2003) demonstraram a fraca convergência entre escalas de IE de desempenho e as de auto-relato, tendo sido observadas correlações de 0,18 e 0,21 entre o MSCEIT e o SSRI (Schutte et al., 1998) e o MSCEIT e o E-QI (Bar-On, 1997), respectivamente. O SSRI e o E-QI são medidas de auto-relato.
Além disso, outro aspecto problemático importante referente ao uso de medidas de auto-relato corresponde à existência na literatura de altas correlações entre escalas que medem IE através de auto-relato e escalas de personalidade (Brackett \& Mayer, 2003; Dawda \& Hart, 2000; Saklofske, Austin, \& Miniski, 2003; Salovey, Mayer, Caruso, \& Lopes, 2001; Schutte et al., 2001; Van der Zee, Thijs, \& Schackel, 2002). Um estudo mais atual, realizado por Bastian, Burns e Nettelbeck (2005), demonstrou que escalas de auto-relato de IE se relacionam mais com escalas de personalidade do que com medidas de IE baseadas em desempenho. A escala de auto-relato TTMS (Trait Meta Mood Scale $)$ correlacionou-se significativamente $(p<0,01)$ com Neuroticismo $(r=-0,42)$, Extroversão $(r=0,62)$, Abertura $(r=0,44)$, Socialização $(r=0,31)$, Realização $(r=0,32)$ e Satisfação de vida $(r=0,51)$. Enquanto isso, o MSCEIT apenas apresentou correlações significativas ( $p$ $<0,01)$ com Abertura $(r=0,23)$ e Socialização $(r=0,19)$. Além disso, as escalas de habilidades cognitivas apresentaram correlações mais fortes com a escala de IE baseada em desempenho (Brackett \& Mayer, 2003; O’Connor \& Little, 2003). O TTMS não apresentou correlação significativa com o Matrizes Progressivas de Raven e o MSCEIT se correlacionou significativamente $(p<0,01)$ com o Raven, porém de forma moderada $(r=0,27)$, o que permite inferir que esta escala se relaciona com medidas de inteligência, porém distingue-se da inteligência geral padronizada. Outra pesquisa conduzida por Woyciekoski (2006) obteve correlações significativas $(p<0,01)$ entre a escala de autorelato MIE e as dimensões Neuroticismo $(r=-0,39)$ e Extroversão $(r=0,50)$.

Estes achados permitem inferir que o MSCEIT possui validade discriminante porque fornece informações sobre diferenças individuais, as quais não estão incluídas nas medidas dos Cinco Grandes Fatores (CGF) e de desempenho acadêmico (Brackett, Mayer, \& Warner, 2004). Conforme Lopes et al. (2003) e Roberts, Zeidner e Matthews (2001), a evidência de validade discriminante em relação a construtos de personalidade é consistente com pesquisas atuais: estudos apontam que as correlações do MEIS ou MSCEIT e medidas de traços de personalidade raramente superam 0,30. Em contrapartida, estes resultados levaram alguns pesquisadores a sugerir que escalas de auto-relato talvez fossem mais bem caracterizadas como inventários de personalidade do que medidas de IE (Mayer, Caruso, et al., 2000).

Todavia, apesar dos problemas referentes às propriedades psicométricas das escalas de auto-relato, alguns autores têm defendido que elas poderiam ser válidas no âmbito da pesquisa, na medida em que possibilitariam a investigação da auto-percepção (Rooy \& Viswesvaran, 2004; Zeidner, Shani-Zinovich, Matthews, \& Roberts, 2005) da IE em contraste com a competência, uma questão, que de acordo com Zeidner et al. (2005), também seria relevante para o campo de estudo da inteligência tradicional. Da mesma forma, Petrides e Furnham (2000, 2001) propuseram que é necessário fazer uma distinção entre IE 
de Traço e habilidade de IE. Segundo os autores, ambos constituem construtos distintos, e não formas diferentes de medir o mesmo construto. $\mathrm{O}$ primeiro incluiria disposições comportamentais e auto-percepção de habilidades e deveria ser medido por meio de questionários de auto-relato, ao passo que o segundo refere-se a habilidades propriamente ditas e deveria ser medido por meio de instrumentos objetivos, ou quasi-objetivos, que requeiram o desempenho. Um estudo realizado por O'Connor e Little (2003), corroborou esta teoria. $\mathrm{O}$ padrão de correlações observadas entre o MSCEIT e o EQ-I se mostrou inconsistente para instrumentos que medem o mesmo construto, tendo o EQ-I se correlacionado significativamente $(p<0,01)$ com dimensões da personalidade (Extroversão $r=0,33$; Ansiedade $r=$ "0,76 e Independência $r=0,43$; medidas pelo 16PF) e não se correlacionado significativamente com habilidade cognitiva. Padrões opostos foram obtidos com o MSCEIT.

Por fim, conforme já fora exposto anteriormente, medidas de inteligência que abrangem a inteligência verbal e de desempenho foram desenvolvidas ao longo do último século até o presente momento. Apesar de progressos na construção destas escalas, também tem sido evidente a insatisfação por parte de muitos pesquisadores acerca de concepções limitadas das capacidades mentais. Sendo assim, muitos têm proposto modelos mais amplos para as capacidades mentais (Gardner, 1995; Guilford, 1967; Mayer, Caruso, et al., 2000; Sternberg, 1988; Thorndike, 1936). A IE inscreve-se com a proposta de ampliar o campo de habilidades no qual a inteligência geral está baseada. Conforme Mayer, Caruso, et al. (2000): "talvez uma inteligência geral que incluir a IE possa constituir um preditor mais poderoso de importantes capacidades humanas do que uma inteligência que não a inclua" (p. 295).

\section{Validade Preditiva da IE}

A relevância da IE depende da sua capacidade de predizer importantes aspectos da vida do homem, incluindo o comportamento. Sendo assim, psicólogos, educadores, empresários e pesquisadores têm procurado conhecer as implicações desta forma de inteligência na vida das pessoas, bem como se interessado nas aplicações práticas da IE nos contextos educacional, ocupacional e clínico (Salovey, Woolery, \& Mayer, 2001). Muitas perguntas têm sido levantadas, tais como: qual a relação da IE com o sucesso acadêmico e profissional, como a IE afeta as relações interpessoais, se pessoas com altos níveis de IE comportam-se diferentemente de pessoas com baixos níveis, entre outras (Brackett et al., 2004; Matthews et al., 2002).

Contudo, não está muito claro ainda o que a IE prediz. Alguns estudos preliminares sugeriram que baixos escores em IE estariam relacionados ao uso de álcool, cigarro e drogas ilegais, comportamento desviante e auto-destrutivo, interações pobres (pouco significativas e sem profundidade) com amigos, além de sentimentos de impotência (Brackett \& Mayer, 2003; Trinidad \& Johnson, 2001). Enquanto isso, altos níveis de IE foram relacionados à qualidade das re- lações e interações sociais, a habilidades sociais e comportamento próssocial, relações familiares positivas, maiores níveis de otimismo e satisfação de vida (Brackett, Rivers, Shiffman, Lerner, \& Salovey, 2006; Mayer, Caruso, et al., 2000; Salovey, Mayer, et al., 2001). Além disso, escalas de IE de desempenho também têm sido relacionadas a gerenciamento de estresse, desempenho acadêmico e comunicação efetiva (Brackett \& Mayer, 2003; Brackett \& Salovey, 2004; Mayer, Salovey, \& Caruso, 2004b). Mayer et al. (2004a) postularam que pessoas com altos escores em IE seriam mais aptas para perceber as emoções, utilizá-las para produzir pensamento, compreender seus significados, além de controlar as emoções de uma forma mais eficaz do que os outros. Sobretudo, a capacidade de resolver problemas de ordem emocional, nessas pessoas requeriria menos esforço cognitivo (Reis et al., 2007). Além disso, pessoas emocionalmente inteligentes frequentemente apresentariam escores altos em inteligência verbal, social, além de outras inteligências (especialmente se a capacidade de compreensão das emoções também fosse alta), e tenderiam a ser mais abertas a novas experiências e mais sociáveis. Estas pessoas se inclinariam mais a trabalhos que envolvem interações sociais do que a profissões que requerem atividades administrativas e financeiras. Pessoas com altos níveis de IE, também seriam pessoas que tenderiam a estar mais aptas para identificar e reportar suas metas, conquistas e objetivos. Abaixo, é apresentado e discutido o potencial de aplicação da IE, além de suas contribuições para resolução de aspectos práticos da vida.

\section{Satisfação de Vida}

Conforme Matthews et al. (2002), cada vez mais psicólogos têm apontado que a capacidade de compreensão das emoções em si e nos outros, constitui um aspecto crucial para uma vida satisfatória. Pessoas auto-conscientes e sensíveis aos demais teriam maiores habilidades para administrar suas questões de forma mais sábia e adequada ao contexto, mesmo quando em situações adversas. Por outro lado, pessoas incapazes de reconhecer as próprias emoções e as alheias tenderiam a vivenciar problemas como desentendimentos constantes, frustrações e fracassos relacionais.

Foram conduzidas pesquisas que investigaram as relações entre satisfação de vida e IE (por meio de medidas de auto-relato e de desempenho), tendo sido reportadas correlações positivas de baixas a moderadas, na ordem de $r=$ 0,11 a $r=0,61$ (Ciarrochi, Chan, \& Caputi, 2000; Gannon \& Ranzijn, 2005; Mayer, Caruso, et al., 2000; Palmer, Donaldson, \& Stough, 2002; Saklofske et al., 2003; Schutte, Lopes, \& Malouff, 2000). Além disso, foram encontradas evidências de que a habilidade de Gerenciamento Emocional prediz satisfação em relacionamentos, de forma mais significativa do que as dimensões de personalidade (Lopes et al., 2003). Outro estudo conduzido por Bastian et al. (2005) (onde foram utilizadas as escalas TMMS e MSCEIT), demonstrou que indivíduos com altos escores em IE apresentaram maior satisfação de vida, capacidade de resolu- 
Woyciekoski, C. \& Hutz, C. S. (2009). Inteligência Emocional: Teoria, Pesquisa, Medida, Aplicações e Controvérsias.

ção de problemas, habilidade de coping, além de menores níveis de ansiedade.

\section{Contexto Clínico}

Tem sido afirmado que a IE pode apresentar contribuições importantes no setting clínico (Matthews et al., 2002). Isso porque ela seria um elemento fundamental para a ocorrência de mudança num processo terapêutico; a qual envolveria necessariamente alguma capacidade da pessoa em identificar, regular, controlar ou modificar suas emoções e pensamentos produzidos inicialmente por estas. Ou seja, a capacidade de compreender e analisar as emoções próprias e alheias culminaria numa melhor compreensão da relação da pessoa com os outros e com o ambiente que lhe cerca, o que por sua vez, poderia promover regulação emocional mais adaptada, além de maior bem-estar (Salovey, Bedell, Detweiler, \& Mayer, 2000). Pessoas com altos níveis de IE poderiam obter maior aproveitamento de um processo terapêutico, bem como talvez pudessem apresentar menores índices de desistência ou abandono da terapia. Salovey et al. (2000) afirmaram que a capacidade de reconhecer as emoções e modificar certos pensamentos que ocasionam reações emocionais insatisfatórias constitui um dos objetivos centrais de um tratamento. Portanto, uma terapia envolveria uma aprendizagem por parte do paciente, sobre como discriminar suas emoções, bem como as dos outros.

Além disso, sabe-se que a grande maioria de sujeitos portadores de desordens clínicas e mentais, demonstram deficiências no processamento da informação emocional e na capacidade de auto-controle, as quais provavelmente seriam deficientes em IE (Matthews et al., 2002). Por outro lado, indivíduos com altos níveis de IE apresentariam uma maior abertura emocional, menos pensamento ruminativo e maior suporte social (Salovey et al., 2000). Pessoas que percebem e entendem as emoções alheias podem estar mais capacitadas para responder ao seu ambiente social, e, portanto, possuir uma rede social mais sólida, o que os tornaria menos vulneráveis a doenças emocionais (Matthews et al., 2002). Em relação à personalidade, foram observadas correlações com as dimensões dos CGF, as quais apontam algumas características do indivíduo emocionalmente inteligente. Pessoas com altos níveis de IE tendem a ser altas em Socialização $(r=0,21)$, Abertura $(r=0,17)$ e Realização $(r=0,11)$. Estes resultados constituem as médias das correlações obtidas em cinco estudos entre IE (medida pelo MEIS e MSCEIT) e os CGF (Mayer et al., 2004a).

Mayer, Salovey, et al. (2000) também sugeriram o uso de testes de IE em procedimentos psicodiagnósticos, na medida em que estas escalas poderiam fornecer informações úteis sobre os recursos emocionais de clientes, as estratégias emocionais por eles utilizadas, além de seu potencial para o aproveitamento terapêutico. Os autores visualizaram o uso destas escalas por parte de clínicos, com o objetivo de melhorar a previsão dos progressos terapêuticos de seus clientes. Ainda, estas escalas talvez pudessem ser úteis para seleção de tratamentos específicos nos setores públicos de saúde.

\section{Contexto Escolar}

Um dos aspectos mais importantes do debate atual acerca da IE envolve as suas contribuições para a predição do sucesso escolar e profissional. Embora tenha se afirmado que a IE poderia explicar grande parte do comportamento bem sucedido nestes contextos, poucos estudos têm efetivamente demonstrado estas relações de forma empírica (Mayer, Caruso, et al., 2000). Em relação ao desempenho acadêmico, Zeidner et al. (2005) conduziram uma pesquisa com estudantes de segundo grau, na qual alunos considerados "A" (gifted) obtiveram escores mais altos no MSCEIT, e as diferenças entre grupos variaram estatisticamente de acordo com as diferenças no teste de vocabulário. Um dado curioso desta pesquisa refere-se ao fato que esses mesmos alunos selecionados por aptidão acadêmica também demonstraram uma falta de conhecimento ou insegurança em relação a suas habilidades emocionais (demonstradas pelos seus baixos escores no SSRI, que constitui uma escala de auto-relato de IE, que fornece uma medida da auto-percepção da IE).

Matthews et al. (2002) salientaram uma crescente preocupação por parte de autoridades e educadores com os problemas comportamentais, alguns dos quais tem desencadeado inclusive assassinatos em escolas americanas, cometidos pelos próprios alunos. Estes eventos têm sido atribuídos, por muitos educadores e psicólogos, a uma falha na aprendizagem das habilidades emocionais e sociais dos estudantes, aspectos que compõem a IE. Sendo assim, segundo Mayer e Geher (1996), estes profissionais acreditam que cada vez mais tem se mostrado necessário e pertinente, a consideração de aspectos relativos à aprendizagem de habilidades emocionais no ambiente escolar, o qual deveria também promover esta educação. Para estes autores a IE seria crucial para o sucesso acadêmico e para uma experiência positiva neste contexto. Similarmente, Eisenberg, Cumberland e Spinrad (1998) argumentaram que a IE influencia o desenvolvimento emocional e social das habilidades, que constituem pré-requisitos básicos para a aprendizagem e ajustamento escolar. Além disso, tem sido levantada a hipótese de que é possível educar aqueles que, em virtude de suas experiências, não adquiriram competências emocionais suficientes. Por meio de treinamento eles poderiam desenvolver habilidades para melhor reconhecer seus sentimentos, expressá-los e regulá-los (Mayer \& Geher, 1996).

\section{Contexto Organizacional}

Apesar de que na maioria dos diferentes cargos, a inteligência geral constitua o maior preditor de desempenho profissional e produtividade, não se pode negligenciar o fato de que o valor das pessoas em uma organização não se reduz ao seu desempenho objetivo, ou as suas capacidades técnicas necessárias para a execução de determinado ofício. Também devem ser levadas em conta outras qualida- 
des, não menos essenciais, como integridade, confiança, iniciativa, além das suas habilidades para trabalhar e conviver com outros.

Com base nesta premissa, tem sido apontada uma série de competências emocionais e sociais, as quais constituiriam precursores cruciais do sucesso ocupacional (Cooper \& Sawaf, 1997; Goleman, 1998; Matthews et al., 2002). Estas competências envolveriam: (a) a auto-consciência emocional (insights psicológicos, reconhecimento de emoções e sentimentos próprios e alheios); (b) a capacidade de identificar as necessidades dos outros e responder de forma adequada e (c) a capacidade de regulação emocional. Desta forma, tem-se postulado que a IE apresentaria validade preditiva para uma série de comportamentos organizacionais, em um nível superior ao da inteligência (Cooper \& Sawaf, 1997). Em 1995 e 1998, Goleman afirmou que a IE seria capaz de predizer o sucesso na vida e no trabalho. Embora tais afirmações careçam de bases científicas, seria pertinente reconhecer a IE como um preditor adicional do sucesso organizacional, porque ela influenciaria a habilidade das pessoas em lidar de forma efetiva e adaptada nas situações que envolvessem pressões e demandas ambientais. Similarmente, Salovey et al. (2000) argumentaram que pessoas inteligentes emocionalmente deveriam ser mais bem sucedidas ao responder a situações estressantes, porque elas seriam mais capazes de avaliar as suas emoções e, portanto, regulá-las. Além disso, a capacidade de compreender as pessoas constituiria um aspecto importante de qualquer gerenciamento efetivo (Goleman, 1996, 1998). Embora o gerenciamento de pessoas envolvesse capacidades técnicas, ele envolveria também as emocionais; e a posse de ambas habilidades poderia fornecer informações relevantes para otimizar a execução dos trabalhos organizacionais (Matthews et al., 2002).

Da mesma forma, Mayer, Salovey, et al. (2000) sugeriram que a IE poderia contribuir com os comportamentos organizacionais, especialmente ao que se refere ao desempenho do indivíduo no seu trabalho, bem como às relações interpessoais estabelecidas neste ambiente. Esta preocupação é pertinente na medida em que os ambientes organizacionais são caracterizados por relacionamentos interpessoais e pela presença freqüente de conflito. Sendo assim, Day e Carroll (2004) afirmaram que o sucesso no trabalho envolveria, além do trabalho propriamente dito ou desempenho da tarefa bem sucedido, o desempenho de outros comportamentos considerados relevantes para um grupo ou organização. Já em 1964, Katz (citado por Matthews et al., 2002) havia referido que o sucesso no trabalho seria também determinado por comportamentos que promovessem a ajuda mútua entre colegas e a própria instituição, criando um clima organizacional positivo. Mayer, Salovey, et al. (2000) propuseram que as pessoas emocionalmente inteligentes estariam aptas a identificar o estado emocional de seus colegas, clientes e superiores, além de responder de forma apropriada as suas percepções. Elas seriam mais hábeis para se engajar em grupos e promover cooperação. Também tenderiam a ser empáticas (Ciarrochi et al., 2000) e, portanto, segundo Abraham (1999), mais inclinadas a adotar a visão da organização e agir de forma benéfica para a empresa. Giles (2001, citado por Mayer, Salovey, et al., 2000) encontrou correlações positivas entre aspectos do envolvimento organizacional de subordinados e a IE de supervisores. Igualmente, relações com clientes poderiam sofrer a influência positiva da IE.

Além disso, tem sido observado o uso de testes de IE em seleção de pessoal, especialmente nas organizações ocidentais. Isso porque, cada vez mais, as empresas estão reconhecendo a relevância das habilidades relativas à IE, as quais constituiriam, de acordo com alguns autores, elementos vitais para o bom funcionamento de uma organização. Numa pesquisa, foram obtidas fortes correlações entre IE e desempenho organizacional (Multi-Health Systems, 2001, citado por Day \& Carroll, 2004). Devido a estes e outros apontamentos, tem se observado um aumento significativo de programas delineados para promoção de habilidades e competências emocionais no contexto organizacional. Contudo, Day e Carroll (2004) sugeriram que é necessária cautela nas afirmações sobre o poder de predição da IE, além de mais estudos que estabeleçam a validade deste tipo de inteligência, de modo mais consistente. Acima de tudo, é necessário pontuar que há pouca evidência capaz de sustentar as afirmações acima realizadas. Estes achados devem ser considerados como resultados iniciais de um campo promissor em expansão.

\section{Considerações Finais}

Como é possível observar, há uma importante dissonância entre as afirmações feitas em relação ao poder da IE, e a real condição das pesquisas científicas preliminares, as quais têm se mostrado incapazes de sustentar tais afirmações. Contudo, algumas evidências têm sido obtidas, sobre o fato de que alguns testes podem estar medindo a IE de forma fidedigna, embora sejam passíveis de aprimoramento. Os testes mais promissores são as escalas baseadas em desempenho, as quais podem predizer alguns comportamentos importantes, mas que também precisam ser melhor investigadas. Sobretudo, a IE encontra-se no seu período inicial de desenvolvimento, e muito trabalho ainda é necessário, até que ela atinja o status de um construto estabelecido, como a inteligência e as dimensões de personalidade (CGF, por exemplo).

Ao longo deste artigo procurou-se demonstrar que um dos principais aspectos problemáticos relacionados ao campo da IE, refere-se às dificuldades teóricas encontradas em defini-la e, portanto, medi-la. Embora reconheçamos esta deficiência, deve-se ressaltar que o desenvolvimento de uma teoria deste tipo é desafiador, na medida em que abrange um campo conceitual complexo e multidimensional sobre as inter-relações entre emoção e cognição, e das competências daí decorrentes, especialmente o que se refere à capacidade de regulação emocional para adaptação. Conforme pontuado, distintos modelos têm procurado definir e explicar a IE: o modelo cognitivo (Mayer, Caruso, et al., 2000; Mayer, Salovey, et al., 2000) e o modelo misto (Bar-On, 1997; Goleman, 1995/1996; Schutte et al, 1998; 
Woyciekoski, C. \& Hutz, C. S. (2009). Inteligência Emocional: Teoria, Pesquisa, Medida, Aplicações e Controvérsias.

Siqueira et al., 1999). O primeiro postulou que a IE deve ser concebida por meio de habilidades relacionadas à emoção e à cognição, e ser medida por meio de escalas de desempenho. Esta visão é compatível com evidências empíricas, as quais apontam que a inteligência é composta por sistemas complexos de habilidades (Matthews et al., 2002; Sternberg, 1992; Sternberg \& Salter, 1982). Opostamente, o segundo incluiu nas suas definições características não intelectivas, como dimensões da personalidade e postulou que a IE poderia ser medida por meio de questionários de auto-relato. Estes últimos têm sido reportados como apresentando importantes sobreposições com escalas que medem construtos pré-existentes (como personalidade), relegando a IE uma condição de redundância (Roberts et al., 2001).

Como efeito disso, escalas de auto-relato têm apresentado maior validade preditiva do que testes de desempenho (ver Bar-On, 1997). Contudo, esta validade é, sobretudo, consequiência das sobreposições excessivas destas escalas com construtos existentes. Conforme ressaltaram Petrides e Furnham (2001), a distinção daquilo que é considerado unicamente IE dos traços de personalidade é um aspecto crucial para progressos futuros. Segundo apontaram Matthews et al. (2002), a ausência de clareza conceitual nas definições de IE, pode significar que diferentes concepções da IE podem, na realidade, estar produzindo qualidades psicológicas distintas.

Ainda, conforme ressaltaram Matthews et al. (2002), não faz sentido operacionalizar a IE como um construto inteiramente independente de medidas existentes baseadas em habilidades. Instrumentos de auto-relato de IE em geral não se correlacionam com medidas de IE de desempenho, além de não se correlacionarem com escalas de inteligência, permitindo inferir que não podem constituir uma medida de nenhum tipo de inteligência (no caso da IE). Independentemente destas considerações, a IE não deve ser considerada como um campo infrutífero, ou os esforços de seus proponentes devem ser depreciados, pois se trata de um construto complexo, que apresenta problemas conceituais e metodológicos, o que não é incomum na psicologia.

É necessário, porém, avançar no conhecimento desta área, através do desenvolvimento de medidas de desempenho, similares às utilizadas na pesquisa em inteligência. Este aspecto psicométrico é fundamental para que se possa conceber a IE como uma forma de inteligência. Conforme recomendaram Matthews et al. (2002), um teste de IE poderia ser considerado ideal se comportasse quatro critérios: fidedignidade, validade de conteúdo, validade preditiva e validade de construto. No panorama atual observa-se que os maiores progressos ocorreram em relação à fidedignidade, uma vez que escalas de auto-relato e de desempenho demonstram elevada consistência interna. Todavia, a validade de conteúdo e de construto (especialmente a validade convergente e discriminante) ainda é deficiente e necessita mais pesquisa. Aparentemente, apenas o MEIS e o MSCEIT parecem contemplar estes critérios, apesar de que também constituam medidas que necessitam de aprimoramento (Brackett \& Mayer, 2003; Roberts et al., 2001; Zeidner,
Matthews, \& Roberts, 2001), com relação a seus fundamentos teóricos e a sua validade preditiva, até que possam constituir medidas legitimas da IE. Embora a IE (medida por testes de desempenho) correlacione-se em algum nível com testes que medem habilidades verbais, ela apresenta apenas uma modesta correlação com medidas de personalidade dos CGF, por exemplo (Salovey \& Grewal, 2005). Em um estudo conduzido por Brackett e Mayer (2003), o MSCEIT foi o teste de IE que mais se distinguiu de escalas de Bem-Estar e dos CGF, enquanto que as escalas de autorelato utilizadas na pesquisa se correlacionaram mais fortemente com estes testes, apresentando sobreposições substanciais com estes construtos. Isso também explica, como já fora mencionado, o fato de auto-relatos e testes de IE baseados em desempenho apresentarem fracas correlações. Estes padrões de resultado evidenciam que o sistema criado por Mayer, Caruso, et al. (2000) representa o modelo corrente mais satisfatório, porque o MSCEIT constitui um teste designado a medir a IE como um conjunto de habilidades e competências, e não por meio de dimensões da personalidade.

Mesmo que tenham sido feitas promessas acerca das contribuições práticas da IE em diferentes contextos, é necessário assumir que apenas nos encontramos num estágio inicial da pesquisa, e não há evidências plausíveis capazes de justificar o uso de testes de IE em larga escala nos mais diversos contextos. Pouco se sabe sobre a validade preditiva do MSCEIT e de outras medidas de desempenho. Por outro lado, conforme salientaram Matthews et al. (2002), o modelo de Mayer, Salovey e colegas merece ser aplaudido, na medida em que têm sido feitas iniciativas sofisticadas para definir e medir o que se entende por emocionalmente inteligente. Como destacaram, a inteligência tem sido estudada por mais de um século, o tempo suficiente para que possa ter sido acumulado um corpo científico sólido, capaz de compreender melhor a sua estrutura, processos, e mecanismos. Não deve se estranhar, que exatamente as questões específicas levantadas hoje na pesquisa em IE, constituiriam os aspectos por muito tempo discutidos e investigados na pesquisa em inteligência.

O saldo positivo dos esforços até então acumulados refere-se ao fato de a IE enfatizar a noção de que o sucesso e a adaptação na vida diária, nos mais diversos âmbitos (pessoal, interpessoal e profissional) não dependem unicamente da atividade intelectual. Sobretudo, eles são largamente influenciados por outros fatores, como sensibilidade emocional, competências emocionais e sociais, além da capacidade de sentir e pensar de forma integrada de modo a utilizar estas informações para criação de comportamentos estratégicos e resolução de problemas.

\section{Referências}

Abraham, R. (1999). Emotional intelligence in organizations: A conceptualization. Genetic, Social, and General Psychology, 125, 209-215.

American Psychological Association. (1997). Intelligence: Knowns and unknowns. Washington, DC: Author. 
Bastian, V. A., Burns, N. R., \& Nettelbeck, T. (2005). Emotional intelligence predicts life skills, but not as well as personality and cognitive abilities. Personality and Individual Differences, 39, 1135-1145.

Bar-On, R. (1997). The emotional intelligence inventory (EQ-I): Technical manual. Toronto, Canada: Multi-Health Systems.

Brackett, M. A., \& Mayer, J. D. (2003). Convergent, discriminant, and incremental Validity of Competing Measures of Emotional Intelligence. Personality and Social Psychology Bulletin, 9, 1147-1158.

Brackett, M. A., Mayer, J. D., \& Warner, R. M. (2004). Emotional intelligence and its relation to everyday behaviour. Personality and Individual Differences, 36, 1387-1402.

Brackett, M. A., Rivers, S., Shiffman, S., Lerner, N., \& Salovey, P. (2006). Relating emotional abilities to social functioning: A comparison of performance and self-report measures of emotional intelligence. Journal of Personality and Social Psychology, 91, 779-780.

Brackett, M. A., \& Salovey, P. (2004). Measuring emotional intelligence as a mental ability with the Mayer-Salovey-Caruso emotional intelligence test. In G. Geher (Ed.), Measurement of emotional intelligence (pp. 179-194). New York: Nova Science.

Bueno, J. M. H., \& Primi, R. (2003). Inteligência emocional: Um estudo de validade sobre a capacidade de perceber emoções. Psicologia: Reflexão e Crítica, 16, 279-291.

Butcher, J. (1974). La inteligencia humana (J. A. Forteza, Trans.). Madrid, Spain: Marova. (Original work published 1968)

Campione, J. C., Brown, A. L., \& Ferrara, R. A. (1982). Mental retardation and intelligence. In R. J. Sternberg (Ed.), Handbook of human intelligence (pp. 392-492). New York: Cambridge University Press.

Carroll, J. B. (1982). The measurement of intelligence. In R. J. Sternberg (Ed.), Handbook of human intelligence (pp. 29-122). New York: Cambridge University Press.

Ciarrochi, J., Chan, A., \& Caputi, P. (2000). A critical evaluation of the emotional intelligence construct. Personality and Individual Differences, 28, 539-561.

Cooper, R. K., \& Sawaf, A. (1997). Executive EQ: Emotional intelligence in leaders and organizations. New York: Grosset.

Dawda, D., \& Hart, S. D. (2000). Assessing emotional intelligence: Reliability and validity of the Bar-On Emotional Quotient Inventory (EQ-i) in university students. Personality and Individual Differences, 28, 797-812.

Day, A. L., \& Carroll, S. A. (2004). Using an ability-based measure of emotional intelligence to predict individual performance, group performance, and group citizenship behaviours. Personality and Individual Differences, 36, 1443-1458.

Eisenberg, N., Cumberland, A., \& Spinrad, T. L. (1998). Parental socialization of emotion. Psychological Inquiry, 9, 241-273.

Forgas, J. P. (1995). The affect infusion model (AIM): Review and an integrative theory of mood effects on judgment. Psychological Bulletin, 117, 39-66.

Fortes D’Andrea, F. (1996). Desenvolvimento da personalidade: Enfoque psicodinâmico. Rio de Janeiro, RJ: Bertrand Brasil.

Gannon, N., \& Ranzijn, R. (2005). Does emotional intelligence predict unique variance in life satisfaction beyond IQ and personality? Personality and Individual Differences, 38, 13531364.

Garber, J., \& Dodge, K. A. (Eds.). (1991). The development of emotion regulation and dysregulation. Cambridge, UK: Cambridge University Press.

Gardner, H. (1995). Inteligências múltiplas: A teoria na prática (M. A. V. Veronese, Trad.). Porto Alegre, RS: Artes Médicas.
Gohm, C. L., Corser, G. C., \& Dalsky, D. J. (2005). Emotional intelligence under stress: Useful, unnecessary, or irrelevant? Personality and Individual Differences, 39, 1017-1028.

Goleman, D. (1996). Inteligência emocional (M. Santarrita, Trad.). Rio de Janeiro, RJ: Objetiva.

Goleman, D. (1998). Working with emotional intelligence. New York: Bantam Books.

Guilford, J. P. (1967). The nature of human intelligence. New York: McGraw-Hill.

Lopes, P. N., Brackett, M. A., Nezlek, J. B., Schütz, A., \& Salovey, P. (2004). Emotional intelligence and social interaction. Personality and Social Psychology Bulletin, 30, 1018-1034.

Lopes, P. N., Salovey, P., \& Straus, R. (2003). Emotional intelligence, personality, and the perceived quality of social relationships. Personality and Individual Differences, 35, 641-658.

Lyons, J. B., \& Schneider, T. R. (2005). The influence of emotional intelligence on performance. Personality and Individual Differences, 39, 693-703.

Matthews, G., Zeidner, M., \& Roberts, R. D. (2002). Emotional intelligence: Science and myth. Cambridge, MA: Massachusetts Institute of Technology.

Mayer, J. D., Caruso, D. R., \& Salovey, P. (2000). Emotional intelligence meets traditional standarts for an intelligence. Intelligence, 27, 267-298.

Mayer, J. D., DiPaolo, M. T., \& Salovey, P. (1990). Perceiving affective content in ambiguous visual stimuli: A component of emotional intelligence. Journal of Personality Assessment, 54, 772-781.

Mayer, J. D., \& Geher, G. (1996). Emotional Intelligence and the identification of emotion. Intelligence, 22, 89-113.

Mayer, J. D., \& Salovey, P. (1997). What is emotional intelligence? In P. Salovey \& D. Sluyter (Eds.), Emotional development and emotional intelligence: Implications for educators (pp. 3-31). New York: Basic Books.

Mayer, J. D., Salovey, P., \& Caruso, D. R. (2000). Models of emotional intelligence. In R. J. Sternberg (Ed.), Handbook of intelligence (pp. 396-420). New York: Cambridge University Press.

Mayer, J. D., Salovey, P., \& Caruso, D. R. (2002). Mayer-SaloveyCaruso Emotional Intelligence Test (MSCEIT) user's manual. Toronto, Canada: MHS.

Mayer, J. D., Salovey, P., \& Caruso, D. R. (2004a). A further consideration of the issues of emotional intelligence. Psychological Inquiry, 15, 249-255.

Mayer, J. D., Salovey, P., \& Caruso, D. R. (2004b). Emotional intelligence: Theory, findings, and implications. Psychological Inquiry, 15(3), 197-215.

Mayer, J. D., Salovey, P., Caruso, D. R., \& Sitarenios, G. (2001). Emotional intelligence as a standard intelligence. Emotion, 1, 232-242.

Neisser, U., Boodoo, G., Bouchard, T. J., Boykin, A. W., Brody, N., Ceci, S. J., et al. (1996). Intelligence: Knowns and unknowns. American Psychologist, 51, 77-101.

O'Connor, R. M., Jr., \& Little, I. S. (2003). Revisiting the predictive validity of emotional intelligence: Self-report versus abilitybased measures. Personalility and Individual Differences, 35, 1893-1902.

Palmer, B., Donaldson, C., \& Stough, C. (2002). Emotional intelligence and life satisfaction. Personality and Individual Differences, 33, 1091-1100.

Petrides, K. V., \& Furnham, A. (2000). On the dimensional structure of emotional intelligence. Personality and Individual Differences, 29, 313-320. 
Woyciekoski, C. \& Hutz, C. S. (2009). Inteligência Emocional: Teoria, Pesquisa, Medida, Aplicações e Controvérsias.

Petrides, K. V., \& Furnham, A. (2001). Trait emotional intelligence: Psychometric investigation with reference to established trait taxonomies. European Journal or Personality, $15,425-448$.

Reis, D. L., Brackett, M. A., Shamosh, N. A., Kiehl, K. A., Salovey, S., \& Gray, J. R. (2007). Emotional intelligence predicts individual differences in social exchange reasoning. Neuroimage, 35(3), 1385-1391.

Roberts, R. D., Zeidner, M., \& Matthews, G. (2001). Does emotional intelligence meet traditional standards for an intelligence? Emotion, 1, 196-231.

Rooy, D. L. V., \& Viswesvaran, C. (2004). Emotional intelligence: A meta-analytic investigation of predictive validity and nomological net. Journal of Vocational Behavior, 65, 71-95.

Saklofske, D. H., Austin, E. J., \& Miniski, P. S. (2003). Factor structure and validity of a trait emotional intelligence measure. Personality and Individual Differences, 34, 707-721.

Salovey, P., Bedell, B. T., Detweiler, J. B., \& Mayer, J. D. (2000). Current directions in emotional intelligence research. In M. Lewis \& J. M. Haviland-Jones (Eds.), Handbook of emotions (2nd ed., pp. 504-520). New York: Guilford Press.

Salovey, P., \& Grewal, D. (2005). The science of emotional intelligence. Current Directions in Psychological Science, 14, 281-285.

Salovey, P., \& Mayer, J. D. (1990). Emotional intelligence. Imagination, Cognition and Personality, 9, 185-211.

Salovey, P., Mayer, J. D., Caruso, D., \& Lopes, P. N. (2001). Measuring emotional intelligence as a set of mental abilities with the MSCEIT. In S. J. Lopes \& C. R. Snyder (Eds.), Handbook of positive psychology assessment. Washington, DC: American Psychological Association.

Salovey, P., Woolery, A., \& Mayer, J. D. (2001). Emotional intelligence: Conceptualization and measurement. In G. Fletcher \& M. S. Clark (Eds.), The Blackwell handbook of social psychology: Vol. 2. Interpersonal processes (pp. 279307). Oxford, UK: Blackwell.

Schwarz, N. (1990). Feelings as information: Informational and motivational functions of affective states. In E. T. Higgins \& E. M. Sorrentino (Eds.), Handbook of motivation and cognition (Vol. 2, pp. 527-561). New York: Guilford.

Schutte, N. S., Lopez, E., \& Malouff, J. (2000). The relationship between cognitive intelligence and emotional intelligence assessed as a maximal performance and typical performance. Paper presented at the Models of Intelligence Conference, New Haven, CT.

Schutte, N. S., Malouff, J. M., Bobik, C., Coston, T. D., Greeson, C., Jedlicka, C., et al. (2001). Emotional intelligence and interpersonal relations. Journal of Social Psychology, 141, 523-536.

Schutte, N. S., Malouff, J. M., Hall, L.E., Haggerty, D. J., Cooper, J. T., Golden, C. J., et al. (1998). Development and validation of a measure of emotional intelligence. Personality and Individual Differences, 25, 167-177.

Siqueira, M. M. M., Barbosa, N. C., \& Alves, M. T. (1999). Construção e validação fatorial de uma Medida de Inteligência Emocional. Psicologia: Teoria e Pesquisa, 15, 143-152.

Smith, C. A., \& Lazarus, R. S. (1990). Emotion and adaptation. In L. A. Pervin (Ed.), Handbook of personality: Theory and research (pp. 609-637). New York: Guiford Press.

Sternberg, R. J. (1988). Explaining away intelligence: A reply to Howe. British Journal f Psychology, 79, 509-533.

Sternberg, R. J. (1992). As capacidades intelectuais humanas: Uma abordagem em processamento de informações. Porto Alegre, RS: Artes Médicas.
Sternberg, R. J. (1997). The concept of intelligence and its role in lifelong learning and sucess. American Psychologist, 52, 1030-1045.

Sternberg, R. J., \& Salter, W. (1982). Conceptions of intelligence. In R. J. Sternberg (Ed.), Handbook of human intelligence (pp. 3-28). New York: Cambridge University Press.

Thorndike, R. L. (1936). Factor analysis of social and abstract intelligence. Journal of Educational Psychology, 27, 231-233.

Trinidad, D. R., \& Johnson, C. A. (2001). The association between emotional intelligence and early adolescent tobacco and alcohol use. Personality and Individual Differences, 32, 95-105.

Van der Zee, K., Thijs, M., \& Schakel, L. (2002). The relationship of emotional intelligence with academic intelligence and the Big Five. European Journal of Personality, 16, 103-125.

Woyciekoski, C. (2006). Instrumentos de inteligência emocional de auto-relato medem alguma coisa que instrumentos de personalidade não medem? Dissertação de Mestrado nãopublicada, Instituto de Psicologia, Universidade Federal do Rio Grande do Sul, RS.

Zeidner, M., Matthews, G., \& Roberts, R. D. (2001). Slow down, you move too fast: Emotional Intelligence remains an "elusive" intelligence. Emotion, 1, 265-275.

Zeidner, M., Shani-Zinovich, I., Matthews, G., \& Roberts, R. D. (2005). Assessing emotional intelligence in gifted and nongifted high school students: Outcomes depend on the measure. Intelligence, 33, 369-391.
Recebido: 28/02/2007

$1^{a}$ revisão: 01/10/2007 $2^{a}$ revisão: $17 / 12 / 2007$ Aceite final: 28/02/2008 